\title{
Polynomial Chaos-Kriging metamodel for quantification of the debonding area in large wind turbine blades
}

\author{
Bruna Pavlack ${ }^{1,2}$, Jessé Paixãoㄹ, Samuel da Silva ${ }^{1}$, Americo Cunha Jr², David García Cava ${ }^{3}$
}

\begin{abstract}
This study aims to investigate the performance of a data-driven methodology for quantifying damage based on the use of a metamodel obtained from the Polynomial Chaos-Kriging method (PC-Kriging). The investigation seeks to quantify the severity of the damage, described by a specific type of debonding in a wind turbine blade as a function of a damage index. The damage indexes used are computed using a data-driven vibration Structural Health Monitoring (VSHM) methodology. The blade's debonding damage is introduced artificially, and the blade is excited with an electromechanical actuator that introduces a mechanical impulse causing the impact on the blade. The acceleration responses' vibrations are measured by accelerometers distributed along the trailing and the wind turbine blade. A metamodel is formerly obtained through the PC-Kriging method based on the damage indexes, trained with the blade's healthy condition and four damage conditions, and tested with the other two damage conditions. The PC-Kriging manifests promising results for capturing the proper trend for the severity of the damage as a function of the damage index. This research complements the damage detection analyzes previously performed on the same blade.
\end{abstract}

\section{Keywords}

Structural Health Monitoring, wind turbine blades, damage quantification, damage features, data-driven metamodel, Polynomial Chaos-Kriging

\section{Introduction}

Currently, the society aims for a future where the generation of energy is clearer. Thus, there is an expansion in offshore wind turbines, making studies in this area necessary. Additionally, the maintenance of wind turbine blades involves, in most part, methods based on visual inspection, which can be dangerous and expensive (García and Tcherniak 2019). Therefore, a system for monitoring wind turbines' conditions is of great industrial interest, demanding further development of methodologies for detecting and quantifying damage to these structures (Ciang et al. 2008).

Structural Health Monitoring (SHM) methods are used to diagnose and extract meaningful information about the health from a structure of interest, based on the measured data from sensors distributed and permanently installed along the structure (Larrosa et al. 2014). SHM techniques have four functional levels of the classification proposed by Rytter (1993): Level 1 - damage detection, Level 2 - damage location, Level 3 - damage quantification, and Level 4 - remaining useful life estimate. In the literature, the first three levels are also categorized as diagnosis and the last as prognosis. This work focuses on damage quantification to complement a methodology for damage detection by García and Tcherniak (2019) and thus provide a full damage diagnosis of debonding wind turbine blades. The contribution concerns the quantification of the damage, obtaining the severity by the damage indices, assuming the inherent uncertainties to obtain a robust damage quantification.

The damage detection methodology used in García and Tcherniak (2019) followed the idea of Vibration-based
Structural Health Monitoring (VSHM), which measures vibration responses from a artificial excitation. Thus, the structure's health/current state can be analyzed by monitoring its vibration response changes. The methodology was applied to an SSP $34 \mathrm{~m}$ wind turbine blade. Conventional classification of VSHM methodologies is the division between model-based (Maes et al. 2016) and non-modelbased (or data-driven) methods (Love 2002; AvendañoValencia et al. 2015). In the methodology of this work, a data-driven approach is used. Non-model-based methods depend exclusively on the data measured from the structure under study. These methods also involve the construction of a model, but that model is based on data rather than numerical or analytical models (Avendaño-Valencia et al. 2015). The measurements in the structure's initial state are considered reference states, where these observations can be compared with the structure measurements in operation. Any deviation of the new observations from

${ }^{1}$ UNESP - Universidade Estadual Paulista, Departamento de Engenharia Mecânica, Ilha Solteira, SP, Brasil

${ }^{2}$ IFMS - Instituto Federal de Mato Grosso do Sul, Três Lagoas, MS, Brasil ${ }^{3}$ Universidade do Estado do Rio de Janeiro, Rio de Janeiro, RJ, Brasil

${ }^{4}$ University of Edinburgh, School of Engineering, Institute for Infrastructure and Environment, Scotland, UK

\section{Corresponding author:}

Bruna Pavlack, UNESP - Universidade Estadual Paulista, Departamento de Engenharia Mecânica, Av. Brasil, 56, Ilha Solteira, 15385-000, SP, Brasil.

IFMS - Instituto Federal de Mato Grosso do Sul, Três Lagoas, MS, Brasil Email: bruna.pavlack@ifms.edu.br 
the reference state might be considered an indication of damage. In Tcherniak and Mølgaard (2015), an analysis of an unsupervised methodology was carried out that combined the correlation between the signals measured from different accelerometers. This study demonstrated that methodologies based on data-driven techniques have a big potential for detecting damage on large structures. Currently, many datadriven methodologies are being developed; for example, in Soize and Orcesi (2020) a machine learning approach was presented using only an experimental database, consisting of a small number of records, to detect changes in the rigidity of engineering structures.

The damage quantification is not yet extensively addressed by the scientific community, but it is crucial to improve the safety and useful life of structures, and thus motivating the scientific community to develop some damage quantification methods. Paixão et al. (2020) used AutoRegressive (AR) models on Lamb wave signals measured on a composite material plate to calculate damage-sensitive features for calculating indexes using the Mahalanobis square distance. Quantification was achieved by training a defined curve, using cubic spline functions to predict the delamination area. Da Silva et al. (2019) executed a similar idea when manipulating the possibility of extrapolating these trend curves to future prognostic states when the delamination grows in the same place and with a similar effect. These studies demonstrated that the resources that use AR models are precisely correlated with the structural state and with a smooth tendency that allows the use of cubic spline functions.

Many studies have addressed experiments in wind turbines in the literature, but there is a lack of methodologies to quantify the damage to them. One of the difficulties in quantifying wind turbine blades' damage is that these structures work under variability (temperature, climate, etc.). Therefore, it is of great importance to consider uncertainty quantification. A method with valid results in obtaining uncertainty quantification models is the Polynomial Chaos Expansion (PCE). The PCE method with application in engineering was introduced in Ghanem and Spanos (1990). They proposed a new method using convergent orthogonal expansion for solving problems involving material variability. The material property was modeled as a random field. The results found had a good agreement with the results obtained through a Monte Carlo simulation. In Ghanem and Spanos (1991), the PCE was used to quantify the uncertainty applied to some problems involving mechanical systems. The use of different types of orthogonal polynomials to represent non-Gaussian processes was introduced in Xiu and Karniadakis (2002), which presents a method for solving stochastic differential equations based on Galerkin projections on a polynomial chaos basis. They represented stochastic processes based on an Askey family of orthogonal polynomials, that reduced the dimensionality of the system and led to the exponential convergence of the error. This new methodology, which is reviewed in Xiu (2010), presents satisfactory results in terms of computational cost and precision. One of the significant advantages seen in using the PCE is its rapid convergence and expressing the final solution as a random process and not just as a set of statistics. Bogoevska et al. (2017) mentions that operational structures such as wind turbines have complex dynamic behavior that challenges the applicability of existing SHM strategies for condition evaluation. Thus, Bogoevska et al. (2017) proposes a structure based on the symbiotic treatment of environmental/operational variables acting on the structure's vibration response. A probabilistic model of the PCE was used for uncertainty quantification in the identified structural performance indicators. Avendaño-Valencia et al. (2017) emphasized that effective fatigue monitoring and prediction algorithms for structures such as wind turbines require an accurate representation of their dynamic response in the short and long term scale. The long term can be achieved in a computationally efficient way through the use of metamodels. That article discussed a two-step methodology, in which the first consisted of projecting the Power Spectral Density (PSD) of the measured dynamic response of the wind turbine linearly in an alternative representation space through Principal Component Analysis (PCA). In the second stage, the coefficients of the PCA-based projection were used as a vector of characteristics, represented by a probability density model in the characteristic space, which is associated with environmental/operational variables measured by the Supervisory Control and Data Acquisition (SCADA) of the wind turbine through the PCE. The proposed methodology facilitated the detection of different wind turbine modes, although it can still be used for fatigue simulation and prediction, only by sampling from the resource space. This methodology was demonstrated with real data measured on a wind turbine located in Lübbenau, Germany, measured over three months.

In this study, the methodology discussed for quantifying the size of damage like trailing edge debonding of a wind turbine blade is based on obtaining a metamodel using the Polynomial Chaos-Kriging (PC-Kriging) method. Polynomial Chaos Expansions (PCE) and Kriging are two popular non-intrusive metamodeling techniques (they do not modify or adapt the original model equations). The PCE replaces the computational model with a series of orthonormal polynomials in the input variables, where the polynomials are chosen in coherence with the probability distributions of these input variables (Ghanem and Spanos 1991; Ghanem et al. 2017). The Kriging method assumes that the computational model behaves as a realization of a Gaussian random process whose parameters are estimated from the available computer executions, that is, input vectors and response values (Lataniotis et al. 2015). The PC-Kriging presents itself as a new non-intrusive metamodel approach combining PCE and Kriging. The PCE is close to the computational model's global behavior, while Kriging is responsible for its local variability. Combining these two methods leads to better accuracy, or at least as good, as either method alone (Schöbi et al. 2014; Schöbi et al. 2015). That is why the choice of applying both methods as a combined approach in this work.

Some works were carried out using the combination of the PCE and Kriging methods, and thus it was possible to identify some advantages and disadvantages of this combination. Some of the PC-Kriging method's main advantages are the ease model construction, the low computational cost, the analytical calculation of classical 
statistical measures of the quantity of interest, and its simplicity compared to other machine learning techniques. However, the PC-Kriging is very sensitive to data quality, just like any other machine learning technique, and impossible to apply to large problems, these being some of its disadvantages (Schöbi and Sudret 2014; Du and Leifsson 2020). In Kersaudy et al. (2015), the Specific Absorption Rate (SAR) was evaluated using a surrogate model to reduce the computational cost. Thus, it was considered a sparse representation of the PCE using minimal angle regression as a selection algorithm to retain the most influential polynomials, and the selected polynomials are used as regression functions for the universal Kriging model. This combination proposal was applied to three benchmark examples, and the performances were compared with a standard Kriging model and a sparse PCE classic. The combination of the methods showed an adequate performance. In the literature, some studies used PC-Kriging for quantification problems considering uncertainties. In Schöbi et al. (2016), a new structural reliability method was developed based on the PC-kriging approach, which was coupled to an active learning algorithm known as adaptive Kriging-Monte Carlo Simulation (AK-MCS). The problem was formulated so that the calculation of small probabilities of failure and extreme quantiles were unified. Dubreuil et al. (2018) carried out a parametric study of engineering models under uncertainty, using the PC-Kriging approach. The advantage of the approach developed in this article was the reduction in computational cost, which was demonstrated in several numerical examples and also illustrated in the parametric study of an aircraft wing under uncertainty.

In this study PC-Kriging is used to define a trend curve that associates a damage index with an estimate of the damaged area, considering the uncertainties. Each damage index is calculated based on the data-driven algorithm using the Mahalanobis distance (MD), considering a baseline condition as a reference condition. The work is organized as follows: first, a statement of the problem in question is performed, then the methodologies that will be adopted for the detection and quantification of damage in wind turbine blades are presented. The experiment setup and the introduction of artificial damage, and the data collection procedure are presented. Finally, the results are investigated, and the conclusions have discussed the performances of the proposed VSHM methodology for detecting damage in different locations of the accelerometer and the methodology used to quantify the damage.

\section{Problem statement}

The study presented in this work is carried out on an SSP $34 \mathrm{~m}$ wind turbine blade. The blade is instrumented with 20 triaxial accelerometers, ten along the trailing edge (TE) and ten along the leading edge (LE). The results of this study consist of two parts:

1) Damage detection: the methodology in García and Tcherniak (2019) is used. Such methodology consists of 4 steps: data collection, the reference state, feature extraction, inspection phase, and decision making. Each of these steps is detailed throughout the text. An actuator is used to excite the blade, which is an impact test. An investigation is carried out on the sensitivity of detection and damage progression.

2) Damage quantification: to quantify the damage's size, a metamodel obtained from the PC-Kriging method is used. The metamodel generates a trend curve related to the damage indexes obtained in the detection part with the severity of the damage.

Figure 1 shows a schematic of the entire methodology of this work, with the damage quantification part being the main contribution of this work.

\section{Damage assesssment}

\section{Methodology for damage assessment in wind turbine blades}

The methodology presented in this study is available in García and Tcherniak (2019). This methodology is considered a simple nonparametric method for data compression and information extraction. The procedure is divided into four steps: data collection, the reference state, feature extraction, and inspection phase for decision-making (García and Trendafilova 2014).

\section{Data collection}

The first step is to collect the data from the structure/system. Acceleration signals are measured and discretized into a vector. Each measured signal is first standardized to have zero mean and unit variance and secondly transformed into the frequency domain. Each signal vector realization is arranged in the columns of the matrix $\mathbf{Z}$, i.e.,

$$
\mathbf{Z}=\left(\mathbf{Z}_{1}, \mathbf{Z}_{2}, \ldots, \mathbf{Z}_{M}\right) .
$$

The matrix $\mathbf{Z}$ is constructed from signal vectors obtained on the pristine/healthy state of the wind turbine blade, and it is used for creating the reference state.

\section{Creation of the reference state}

A reference state is created based on the matrix $\mathbf{Z}$, where the observation signal vectors can be compared. First, each vector signal $\mathbf{Z}_{m}$ is embedded into a matrix $\check{\mathbf{Z}}_{m}$ by W-lagged copies of itself. All matrices $\check{\mathbf{Z}}_{m}$ are used to create the full embedded matrix $\check{\mathbf{Z}}$, i.e.,

$$
\check{\mathbf{Z}}=\left(\check{\mathbf{Z}}_{1}, \check{\mathbf{Z}}_{2}, \ldots, \check{\mathbf{Z}}_{M}\right)
$$

The covariance matrix of $\check{\mathbf{Z}}$, which defines the covariance between the different signal vector realizations, is estimated by

$$
\mathbf{C}_{Z}=\frac{\check{\mathbf{Z}}^{t} \check{\mathbf{Z}}}{N} .
$$

The eigendecomposition of $\mathbf{C}_{Z}$ yields $\lambda_{k}$ and $\mathbf{E}^{k}$ eigenvectors in the same order as their corresponding eigenvalues. Each eigenvalue defines the partial variance in the direction of its corresponding eigenvector. A matrix $\mathbf{E}_{Z}$ contains all eigenvectors $\mathbf{E}^{k}$. Each Principal Component (PC) $\mathbf{A}^{k}$ associated with each eigenvector $\mathbf{E}^{k}$ is calculated by projecting the matrix $\check{\mathbf{Z}}$ onto $\mathbf{E}_{Z}$. The Reconstructed Components (RCs) are calculated by convolving the PCs with the associated $\mathbf{E}^{k}$. The RCs are then arranged in 


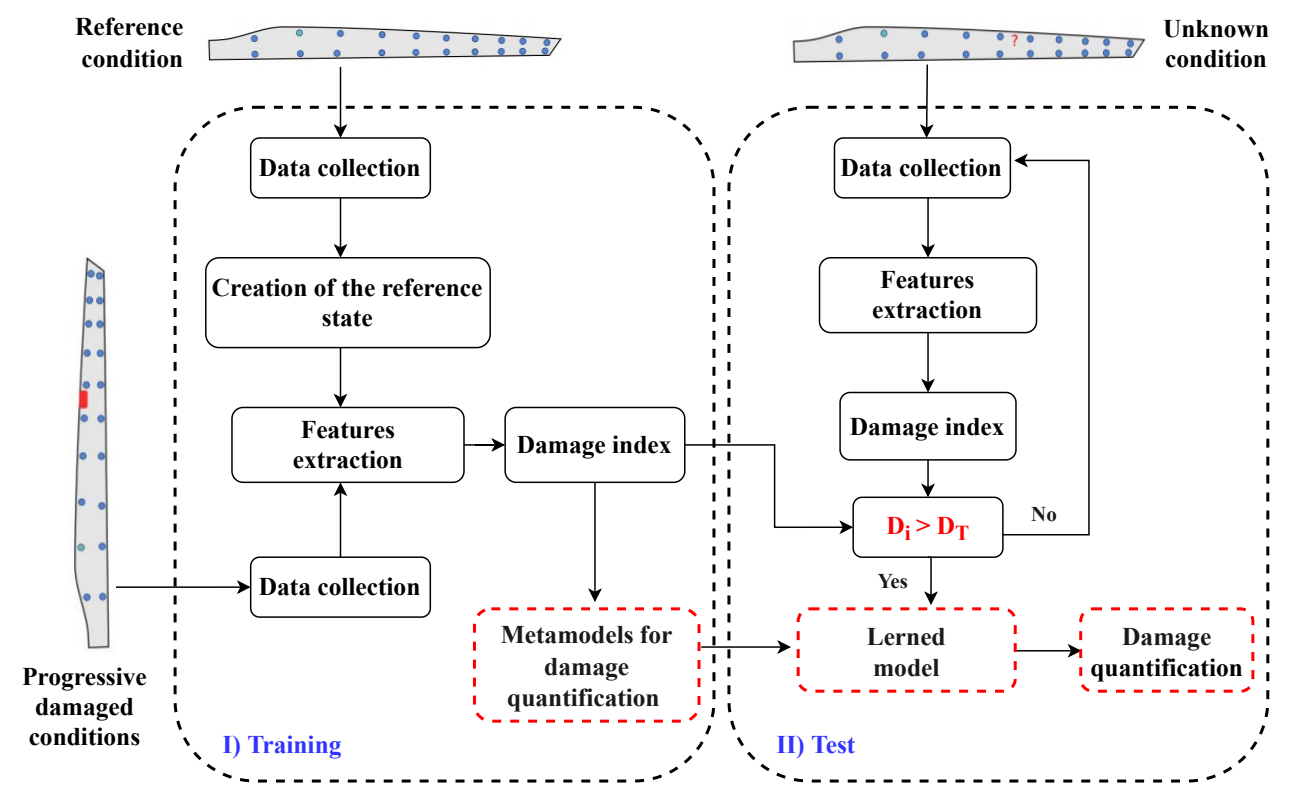

Figure 1. Schematic representation of the proposed methodology for quantifying damage to wind turbine blades.

columns into the matrix $\mathbf{R}$. Therefore, $\mathbf{R}$ can be used as the reference state of the structure/system to which the observation signal vectors are compared.

\section{Feature extraction}

A Feature Vector (FV) is obtained for each new observation signal vector, which will be subjected to damage evaluation by comparing its similarity to the reference state defined by $\mathbf{R}$. An FV is calculated by multiplying an observation signal vector $\mathbf{z}$ with each $\mathrm{RC}$ in the reference state $\mathbf{R}$, as shown in (4) where $j=1, \ldots W$.

$$
T_{j}=\sum_{n=1}^{N} z_{n} R_{n j}
$$

Each $T_{j}$ value represents the inner product between an observation signal vector and each RC. All $T_{j}$ are arranged into a vector $\mathbf{T}$ with dimension $\mathrm{W}$. The FV $\mathbf{T}$ characterizes the observation signal vector onto the feature space.

\section{Inspection phase and decision making}

The baseline feature matrix $\mathbf{T}_{B}$ is created. Once the baseline is defined, an observation FV is then compared with the baseline $\mathbf{T}_{B}$. Using the Mahalanobis distance damage index is obtained,

$$
\mathcal{D}_{i}=\sqrt{\left(\mathbf{T}^{i}-\boldsymbol{\mu}_{B}\right)^{t} \boldsymbol{\Sigma}^{-1}\left(\mathbf{T}^{i}-\boldsymbol{\mu}_{B}\right)},
$$

where $\boldsymbol{\mu}_{B}$ is the mean row of the baseline feature matrix $\mathbf{T}_{B} ; \boldsymbol{\Sigma}$ is its corresponding covariance matrix, and $\mathcal{D}_{i}$ is the damage index.

It is necessary to set a threshold against which damage rates can be assessed to label an observation as an outlier or inlier. A probabilistic threshold $D_{T}$ based on the probability density function (pdf) of the distances measured by the baseline FVs for the baseline matrix is calculated $\mathbf{T}_{B}$. As the damage indexes are always positive $\left(\mathcal{D}_{i}>0\right)$, a lognormal probability density function was used to approximately adjust the data considered as a training set, to define a limit to distinguish between observations of the healthy and damaged structure. The $D_{T}$ limit is calculated by the inverse of the lognormal cumulative density. Thus, the definition of whether or not there is damage is given by the decision below:

$$
\begin{array}{lll}
\mathbf{H}_{\mathbf{0}}: \mathcal{D}_{i} \leq D_{T} & \Rightarrow & \text { Undamage wind turbine blade } \\
\mathbf{H}_{\mathbf{1}}: \mathcal{D}_{i}>D_{T} & \Rightarrow & \text { Damaged wind turbine blade }
\end{array}
$$

\section{Damage quantification methodology}

After detecting the initial trailing edge debonding in the wind turbine blades using a data-driven approach, the user needs to decide if there is an imminent structural failure or if the system can be kept in operation under monitoring to track the damage progression and its impact on structural safety conditions. Therefore, it is imperative to obtain the quantification of the debonding length.

Computer simulation of many problems in modern engineering and applied sciences has a high computational cost. In this context, the metamodelling tries to reduce computational costs and perform sophisticated analyzes, such as reliability analysis and design optimizations (Schöbi et al. 2017). One method for obtaining metamodels is investigated: Polynomial Chaos-Kriging (PC-Kriging). In the next subsection, the method algorithm is presented.

\section{Polynomial Chaos-Kriging}

The PCE method works as a type of "response surface", which locally interpolates the model hypersurface. This method obtains the computational model by sum the orthonormal polynomials to the input variables. This orthogonal expansion decouples stochastic and deterministic objects, that is, the polynomial basis is random and the numerical coefficients are deterministic, obtained from the data. This property made the metamodel construction easier.

In this context, consider a finite-variance computational model $\mathcal{M}: \mathcal{D}_{X} \subset \mathbb{R}^{M} \mapsto \mathbb{R}$, which receives and input a $M$-dimensional vector $X=\left(X_{1}, \cdots, X_{M}\right) \in \mathbb{R}^{M}$ with a given probability density function (PDF) $f_{X}$ defined on the 
support $\mathcal{D}_{x}$, and returns as output the scalar quantity of interest $Y=\mathcal{M}(X) \in \mathbb{R}$, such as illustrated in Figure 2 .

$$
X=\left(X_{1}, \cdots, X_{M}\right) \overbrace{\begin{array}{c}
\text { computational } \\
\text { model }
\end{array}}^{Y=\mathcal{M}(X)}
$$

Figure 2. Computational model under uncertainty.

According to Marelli and Sudret (2015), the Polynomial Chaos Expansion of $\mathcal{M}(X)$ is given by

$$
Y=\mathcal{M}(X) \approx \mathcal{M}^{P C}(X)=\sum_{\alpha \in \mathcal{A}} y_{\alpha} \Psi_{\alpha}(X),
$$

where $\mathcal{A} \subset \mathbb{N}^{M}$ is a subset of the polynomial indexes, $\Psi_{\alpha}(X)$ is a family of orthonormal polynomials with respect to $f_{X}$, and $y_{\alpha}$ are real-valued deterministic coefficients to be determined.

The appropriate family of polynomials is chosen according to the probability distributions of the input variables (Xiu 2010; Ghanem et al. 2017). One of the significant advantages seen in using the PCE is its rapid convergence and expressing the final solution as a random process and not just as a set of statistics.

The Kriging method, also known as Gaussian Process Regression (GPR), is a non-parametric Bayesian approach that has the advantages of working on small data sets and providing measurements that consider the predictions' uncertainties. A Kriging model is called ordinary Kriging when the trend is a single parameter with an unknown value. When the trend is a sum of functions, it is called universal Kriging.

The combination of the Polynomial Chaos Expansion (PCE) and Kriging methods results in the method called Polynomial Chaos-Kriging (PC-Kriging). This combination results in technique metamodeling more accurately than the PCE and Kriging separately. The PC-Kriging uses the PCE type regression to capture the computational model's global behavior and the interpolation type Kriging to capture the variations. The PC-Kriging is considered a universal Kriging technique that obtains the trend from a set of orthonormal polynomials (Schöbi et al. 2014). One of PC-Kriging's main advantages is the ease in building the model and the low computational cost; however, PC-Kriging may not perform well on high-dimensional problems.

The construction of the metamodel by PC-Kriging consists of two stages: (i) the determination of a set of polynomials that defines the trend and (ii) the determination of the ideal correlation parameters and the trend parameters. The polynomials that define the trend are calculated using the PCE by employing the Least Angle Regression (LARS) algorithm. The trend parameters and correlation parts are calculated as in the universal Kriging algorithm by solving the log-likelihood function's maximization using a gradientbased optimization algorithm. These two steps are processed in series, as the set of polynomials can be determined independently of Kriging's settings (Schöbi et al. 2015, 2016; Kersaudy et al. 2015; Schöbi et al. 2017).

The idea of applying PC-Kriging in the damage quantification level is to employ a metamodel to capture the trend between the local damage index and damage size, which has been observed by García and Tcherniak (2019). The local damage index is used as input, and the damage size is used as output for the construction of a PC-Kriging model. The PC-Kriging can be described as

$$
\mathcal{S} \cong \sum_{\alpha \in \mathcal{A}} y_{\alpha} \Psi_{\alpha}\left(\mathcal{D}_{i}\right)+\sigma^{2} \mathcal{Z}\left(\mathcal{D}_{i}, \omega\right)
$$

where $\mathcal{D}_{i} \in \mathbb{R}$ is the local damage index with a given Probability Density Function (PDF) $f_{X}, S$ is the damage size, with $\mathcal{S} \in \mathbb{R}$. The $\sum_{\alpha \in \mathcal{A}} y_{\alpha} \Psi_{\alpha}\left(\mathcal{D}_{i}\right)$ is a weighted sum of orthonormal polynomials that describes the PC-Kriging model trend, where $\Psi_{\alpha}\left(\mathcal{D}_{i}\right)$ are orthonormal polynomials in relation to $f_{X}, \alpha \in \mathcal{A}$ are the indices and $y_{\alpha}$ are the corresponding coefficients; $\sigma^{2}$ is the variance of the process, and $\mathcal{Z}\left(\left(\mathcal{D}_{i}\right), \omega\right)$ is a Gaussian random process with zero mean. $\omega$ describes outcomes of the underlying probability space with a correlation family $\mathcal{R}$ and its hyperparameters $\theta$. That is, the correlation function $\mathcal{R}=\mathcal{R}\left(x, x^{\prime}, \theta\right)$ describes the correlation between two samples of the input space. For example, $x$ and $x^{\prime}$ depends on the hyperparameters $\theta$ (Lataniotis et al. 2015). The PC-Kriging can be interpreted as a universal model of Kriging with a specific trend.

The PC-Kriging algorithm can be done in two ways: sequential and optimal. In sequential PC-Kriging (SPCK), the set of polynomials and the Kriging metamodel are determined sequentially. First, the ideal set of polynomials is determined by the PCE based on LARS. Every set of polynomials is incorporated into the PC-Kriging equation, and then the PC-Kriging metamodel is calibrated as a usual Kriging model, including the calculation of the coefficients $y_{\alpha}$. In the optimal PC-Kriging (OPCK), the model is obtained iteratively. As in SPCK, the ideal set of polynomials is determined by LARS. The LARS algorithm results in a dispersion of the set of polynomials classified according to their correlation with the current residual in each LARS iteration (in decreasing order). Each polynomial is then added individually to the trend of a PC-Kriging model. In each iteration, a new PC-Kriging model is calibrated. At the end of this process, the PC-Kriging models are compared using their leave-one-out (LOO) error estimators. The PCKriging metamodel optimal is chosen according to the one that minimizes the LOO error (Schöbi et al. 2017). The LOO error can be defined as (Schöbi et al. 2015)

$$
\epsilon_{L O O}=\frac{1}{N} \sum_{i=1}^{N}\left(\mathcal{Y}^{(i)}-\mu_{\hat{y},(-i)}\left(\mathcal{X}^{(i)}\right)\right)^{2},
$$

where $\mu_{\hat{y},(-i)}\left(\mathcal{X}^{(i)}\right)$ is the prediction mean $\mu_{\hat{y}}$ of sample $\mathcal{X}^{(i)}$ by a Kriging metamodel based on the experimental design $\mathcal{X}^{(-i)}=\mathcal{X} \backslash \mathcal{X}^{(i)}$ and $\mathcal{Y}=\left\{\mathcal{Y}^{(i)}, i=1, \cdots, N\right\}$ is the exact model response.

In Schöbi et al. (2015), the performances of the Kriging, PCE, SPCK, and OPCK methods, in terms of generalization of relative error in the analytical reference functions, are compared. The results showed that PC-Kriging is better than, or at least as good as, Kriging and PCE methods separately for small experimental projects. Moreover, it was concluded that OPCK is preferable to SPCK because it reduces the number of polynomials in the regression part and, therefore, reduces the metamodel's complexity. Based 
on this conclusion, the OPCK algorithm was employed in this work.

The implementation of the PC-Kriging algorithms was performed using the UQLab*, which is a MATLABbased software framework designed to bring uncertainty quantification (UQ) techniques and algorithms to a broad audience. The UQLab offers an extensive list of algorithms for UQ, including the PC-Kriging method (Marelli and Sudret 2014).

\section{Experimental Application}

The data set used in the experimental application proposed in this work belongs to Bruel \& Kjaer. This data set has been explored recently to validate different methodologies (García and Tcherniak 2019; Ulriksen et al. 2016; Hernandez Crespo 2016). A brief description of the experimental setup will be provided below. A detailed description of the experimental setup can be found in Nielsen et al. (2010).

The experimental application of the methodology proposed was performed in the SSP $34 \mathrm{~m}$ wind turbine blade. The blade was manufactured by SSP-Technology A/S, and the experiments were performed on a test rig at the Wind Energy department, the Technical University of Denmark. The blade of the wind turbine and the experiment's facilities can be seen in Figure 3(b).

The blade was instrumented with 20 triaxial accelerometers, model Bruel \& Kjaer Type 4524-B, positioned as represented in the scheme in Figure 3(a). Ten accelerometers were placed in the trailing edge (TE) and ten in the leading edge (LE). In the experiment, acceleration signals were collected for the blade's impact response under healthy and progressive damaged conditions. Figure 3(c) show the electromechanical actuator used to generate the impact in the structure, and it was placed on the surface outside of the blade at the position indicated in Figure 3(a). In total, 386 signals were collected for seven structural health conditions simulated, being a healthy condition, and six with damage (see Table 1).

\begin{tabular}{ccc}
\hline Condition & Damage Size & Number of Signals \\
\hline H & - & 53 \\
D20 & $20 \mathrm{~cm}$ & 70 \\
D40 & $40 \mathrm{~cm}$ & 61 \\
D60 & $60 \mathrm{~cm}$ & 60 \\
D80 & $80 \mathrm{~cm}$ & 49 \\
D100 & $100 \mathrm{~cm}$ & 54 \\
D120 & $120 \mathrm{~cm}$ & 39 \\
\hline Total & & 386
\end{tabular}

Table 1. Number of signals measured on each experimental test (García and Tcherniak 2019).

One of the main types of damage in wind turbine blades is the adhesive joint debonding (Montesano et al. 2016). This type of damage occurs when an adhesive bond between the laminates of the pressure and suction sides of the blade breaks can happen on both leading and trailing edges. Small debonding size can grow up to a level at which repair is impossible, and the entire blade should be replaced (García and Tcherniak 2019). First, a series of holes through the adhesive between the blade's pressure and suction sides were drilled. Then, using a saw and a chisel, the holes were merged, forming an opening that was gradually extended from $20 \mathrm{~cm}$ up to $120 \mathrm{~cm}$ by increments of $20 \mathrm{~cm}$. The debonded parts were connected by bolts, placed at 10 $\mathrm{cm}$ intervals. The healthy condition was then simulated by tightening all the bolts, and the progressive damaged conditions were reproduced by loosening some bolts. The number of loosened bolts defined the damage size. The location of the damage can be seen in Figure 3(a). More details about the experiment can be found in García and Tcherniak (2019).

\section{Results and Discussions}

This section presents the results of the damage detection and the quantification of the area of the trailing edge debonding. To obtain the results is used the software MATLAB. For damage quantification results is used the toolbox UQLab. It is used to estimate the trailing edge debonding size, using the damage index and optimizing the PC-Kriging model.

\section{Damage detection}

The methodology for damage detection described previously is applied in the dataset of SSP $34 \mathrm{~m}$ wind turbine blade. The data set provides acceleration signals collected from sensors placed in the trailing and leading edges. In García and Tcherniak (2019), the authors defined the parameters chosen for the best methodology performance, which are employed in this work. In the creation of the reference state, it is considered a $M=10$ signal vector realizations with a sliding window size of $W=10$. The feature vector dimension is set to $p=5$. The baseline matrix construction is performed using $s=26$ feature vectors of dimension $p=5$ extracted from healthy condition signals. In the inspection phase, the risk of false alarm probability is set to $\alpha=0.01$ in the lognormal density function. A detailed discussion of each one of these parameters on the methodology performance can be found in García and Tcherniak (2019).

Figure 4 shows the damage index by damage size obtained, considering the accelerometers in the TE to the actuator's position. Figure 5 shows the damage index by damage size obtained, considering the accelerometers in the LE. It can be seen that the accelerometers along the TE detected the damage better than the accelerometers along with the LE.

The damage detection results present three scenarios: i) Damage indexes can detect and track the severity of the damage. This case can be seen in Figure 4, in sensors 2 and 4; ii) The damage is not well detected, as it has many false negatives. However, as the damage increases, there is a tendency for the damage indexes, so the damage's progression is somehow detected. In this case, there is a timedependent feature for damage detection. This can be seen in Figure 4, in sensors 1, 3 and 8, and Figure 5 in sensors 4 and 5; iii) A final scenario is when the damage indexes cannot detect the damage or track its progress. In Figure 4, this can be seen in sensors 6 and 9 and Figure 5, in almost

*https: / /www.uqlab.com/ 


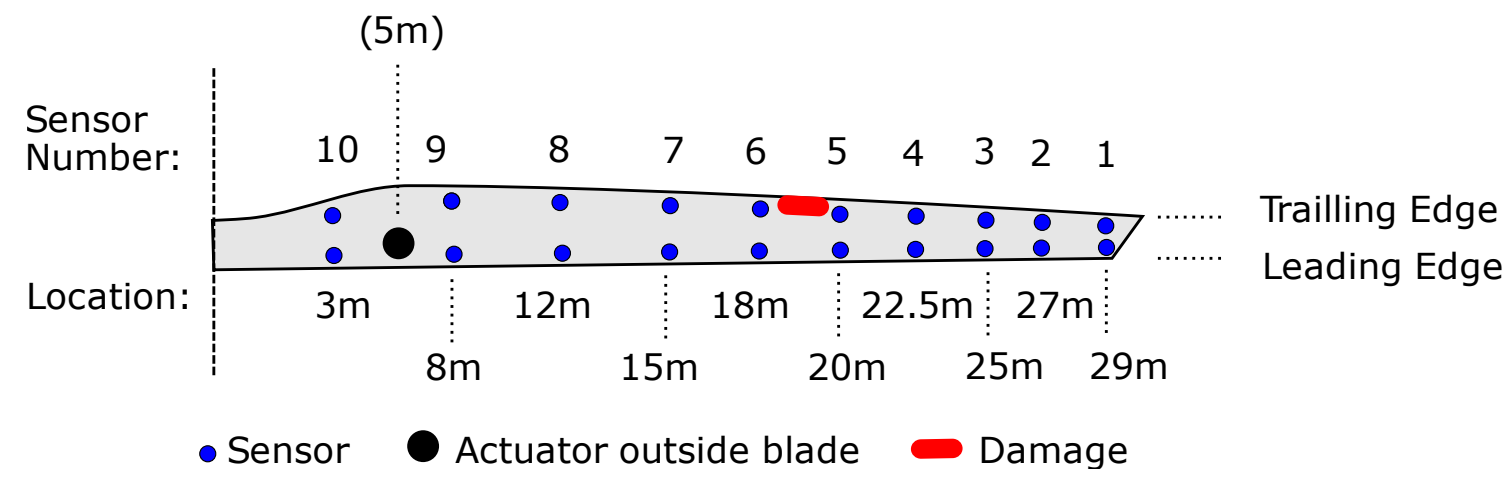

(a) Accelerometers, damage and actuator locations scheme.

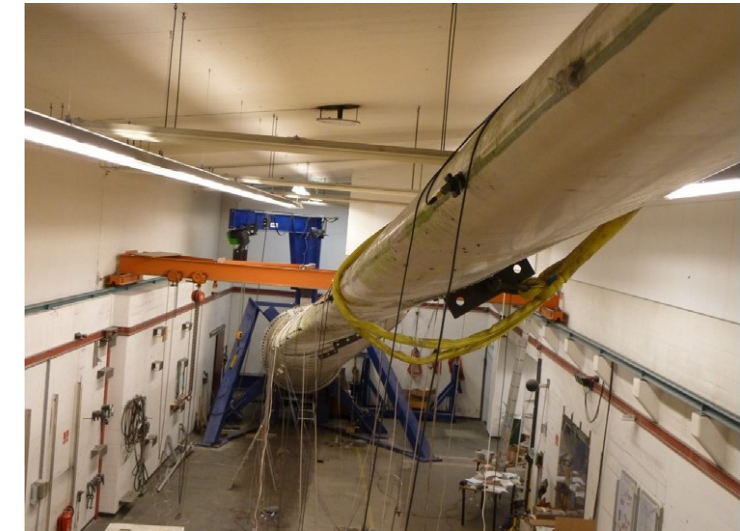

(b) test rig set-up.

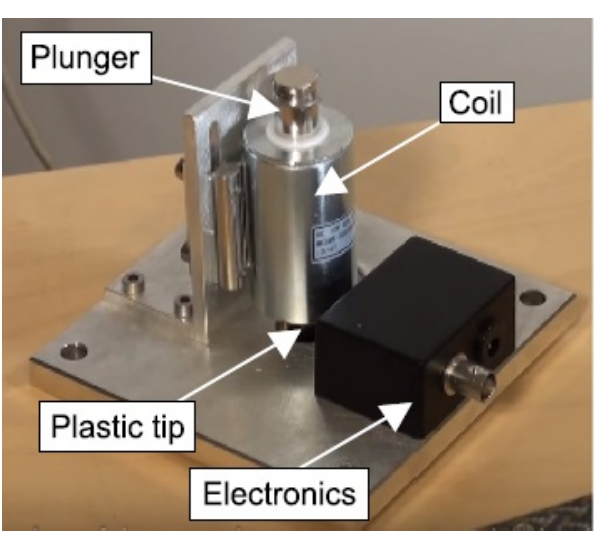

(c) Electromechanical actuator.

Figure 3. Experimental setup of the SSP 34 meters wind turbine blade manufactured by SSP-Technology A/S. The blade was instrumented with 10 accelerometers along the leading edge (LE) and 10 along the trailing edge (TE) and was excited by an electromechanical actuator. The debonding damage was introduced into the blade (García and Tcherniak 2019).
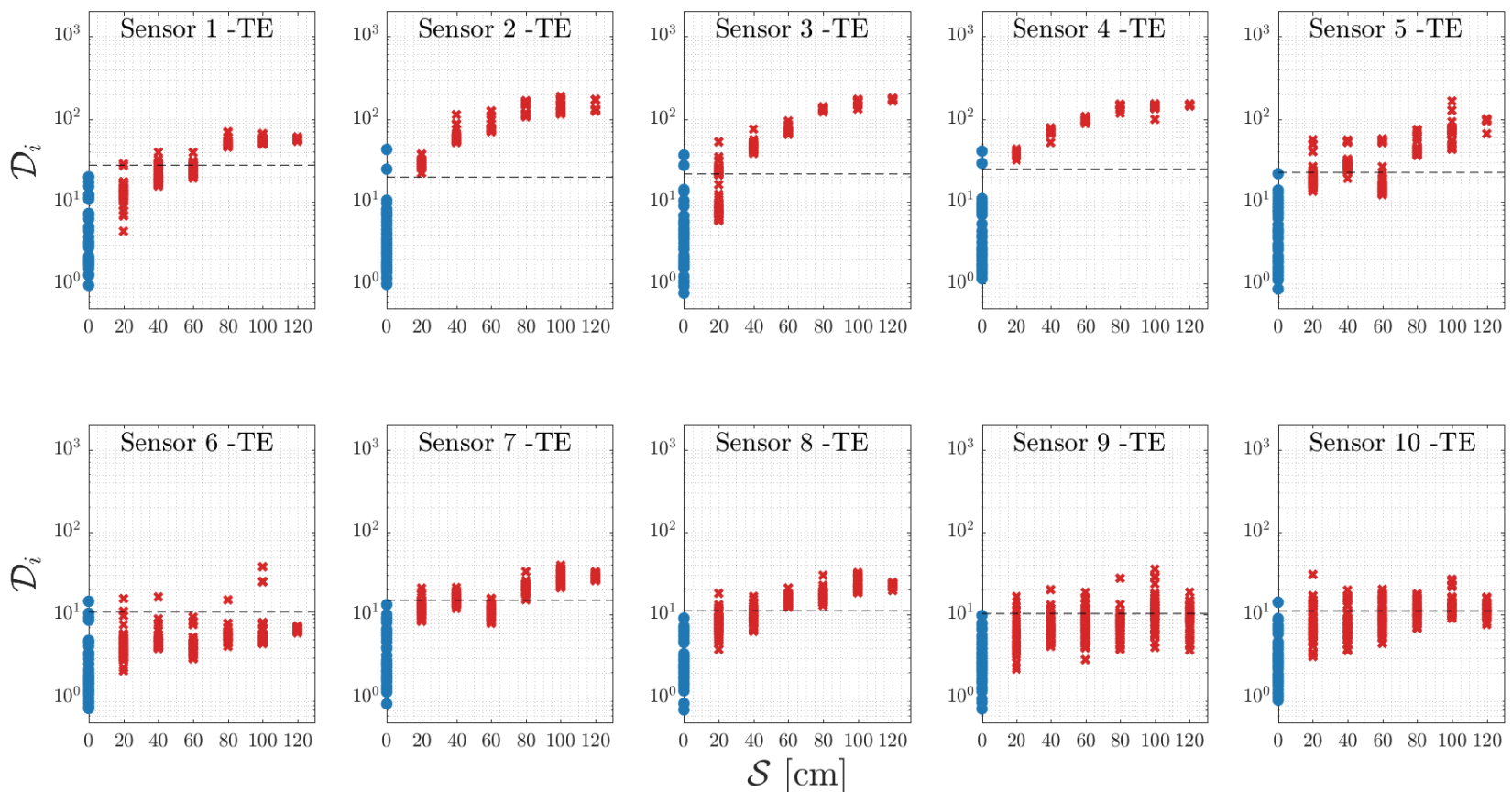

Figure 4. Damage index $\left(\mathcal{D}_{i}\right)$ by damage size $(\mathcal{S})$ for accelerometers in the trailing edge (TE). The damage index in the healthy (O) and damaged (x) conditions The dashed line (_ _ - ) corresponds to the threshold defined by a risk of false alarm probability equal set to $\alpha=0.01$. 

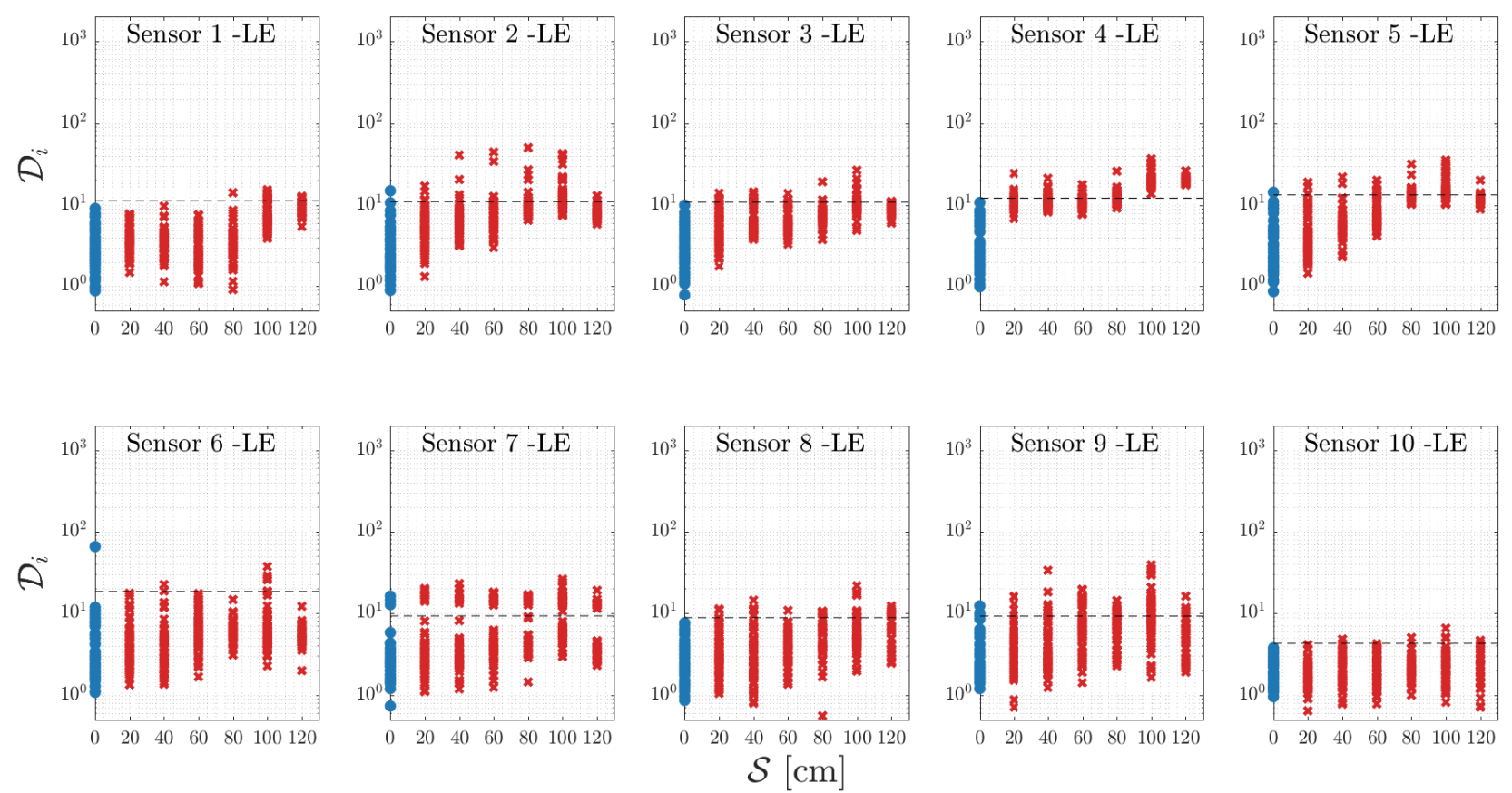

Figure 5. Damage index $\left(\mathcal{D}_{i}\right)$ by damage size $(\mathcal{S})$ for accelerometers in the leading edge (LE). The damage index in the healthy $(\mathrm{O})$ and damaged $(\mathrm{x})$ conditions The dashed line (_ - -) corresponds to the threshold defined by a risk of false alarm probability equal set to $\alpha=0.01$.

all sensors. These results of damage detection influence the results of damage quantification because damage indexes are used.

\section{Damage quantification}

The damage quantification methodology used is based on the construction of a PC-Kriging metamodel, which obtains the trailing edge debonding size as a function of the local damage index. The local damage index is used as input for building a PC-Kriging metamodel. In the model training stage, the following conditions' local damage index is considered: H, D40, D80, D100, and D120. The damaged conditions D20 and D60 are used in the test steps to validate the prediction of damage quantification using metamodel.

In the first stage of the PC-Kriging metamodel construction, the polynomials set that define the trend through the PCE are defined using the LARS algorithm. In the second stage, the ideal correlation parameters and the tendency parameters are calculated the same way they are calculated in the universal Kriging algorithm.

For this work results, an optimal PC-Kriging approach is used; that is, the metamodel is obtained iteratively, and the one chosen is the one that minimized the leave-oneout (LOO) error. The optimal PC-Kriging is chosen because it already has better performance in the literature than the sequential PC-Kriging (Schöbi et al. 2015). Figure 6 shows a schematic representation of the Optimal Polynomial ChaosKriging algorithm. The damage index is always positive, so in the PCE settings, the distribution used is lognormal, and the moments of the PCE settings are the mean and standard deviation of the training data. In the Kriging configuration, a correlation function based on an exponential and ellipsoidal family is used to optimize the maximum likelihood estimate performed by a gradient method to define the GPR model (Lataniotis et al. 2015). The trained models represent a mean and $95 \%$ confidence interval of the predicted distribution. Figure 7 attests to the severity, that is, the size of the trailing edge debonding as a function of the damage index obtained by the PC-Kriging method for the sensors in the TE, and Figure 8 present the results for each sensor in the LE.

Figures 7 and 8 show that most of the damage indexes used in the training and test stages are within the region of the confidence interval, inferring an adequate selection of the training parameters. It is also noted that the PC-Kriging model captured the damage index trend adequately well.

The Root Mean Squared Error (RMSE) metric is chosen to validate the model prediction for test conditions D20 and D60. This metric is frequently used measure of the differences between values estimated by a model or an estimator and the values measured. The RMSE in this work can be defined as

$$
R M S E=\sqrt{\sum_{i=1}^{n} \frac{\left(\mathcal{S}_{e s t}-\mathcal{S}_{m e a}\right)^{2}}{n}},
$$

where $\mathcal{S}_{\text {est }}$ are estimated values for the quantification of the

debonding area, $\mathcal{S}_{\text {mea }}$ are measured values of the debonding area and $n$ is number of observations. The validations of the PC-Kriging model prediction for the test conditions in the TE and LE are presented in Figures 9 and 10. Ideally, the points would concentrate on the diagonal line, as this is where the estimated damage size coincides with the measured damage size. Table 2 presents the RMSE values and the mean of the estimated value for the test conditions (D20 and D60) for each sensor in the TE and LE. 


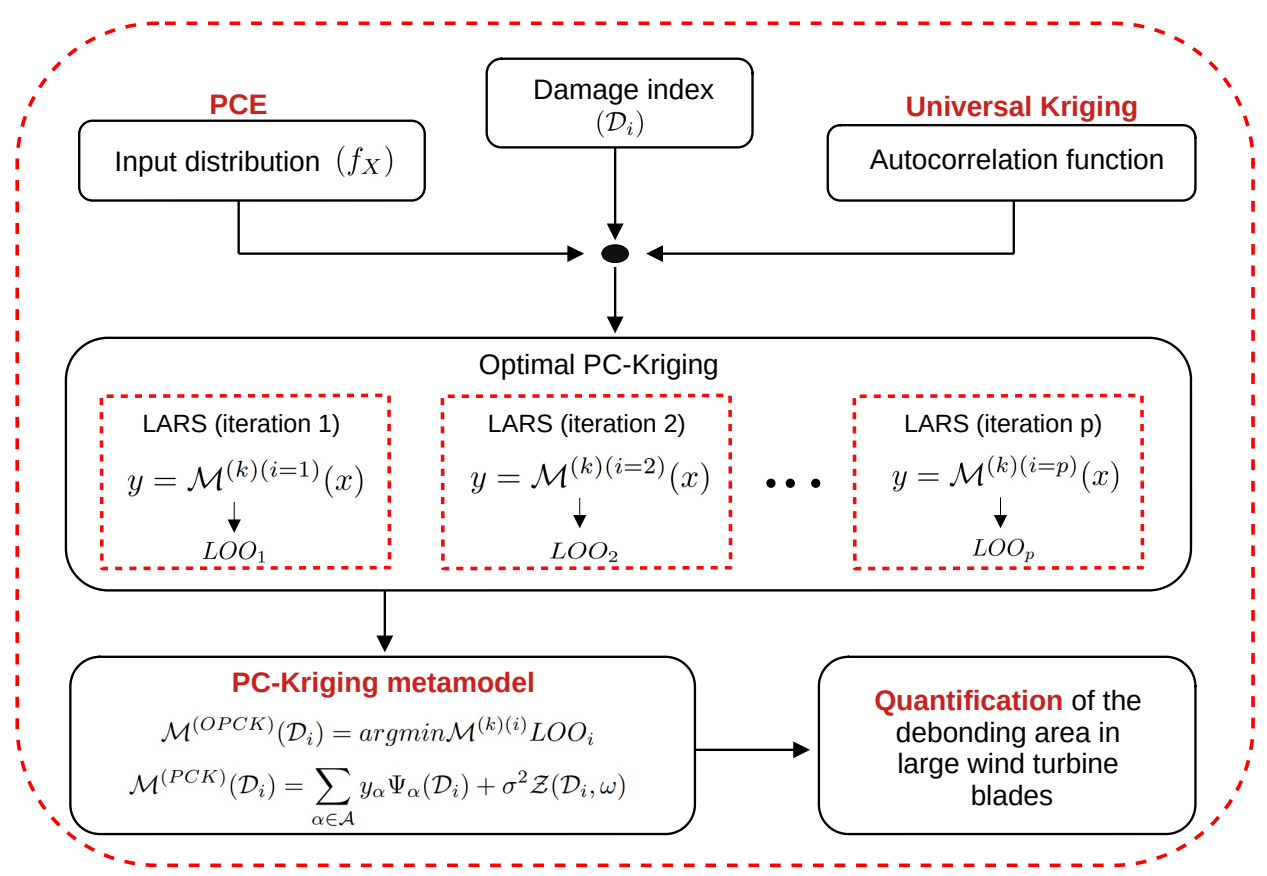

Figure 6. Schematic representation of the Optimal Polynomial Chaos - Kriging algorithm.

Table 2. Value of the root-mean-square error (RMSE) and relative error of the test conditions (D20 and D60) for each sensor along TE and LE by the PC-Kriging metamodel. The sensors that have detected the damage better have the values shown in blue.

\begin{tabular}{cccccccccccc}
\hline & Sensor & 1 & 2 & 3 & 4 & 5 & 6 & 7 & 8 & 9 & 10 \\
\hline \multirow{2}{*}{ TE } & RMSE & 16.8 & 10.0 & 11.4 & 5.83 & 31.1 & 32.0 & 30.6 & 16.4 & 33.6 & 38.5 \\
& D20: Mean est. damage[cm] & 25.81 & 15.58 & 3.31 & 13.99 & 23.31 & 49.04 & 22.89 & 25.14 & 50.40 & 57.52 \\
& D60: Mean est. damage[cm] & 36.77 & 47.95 & 52.25 & 63.54 & 15.78 & 56.23 & 17.86 & 72.04 & 71.38 & 83.89 \\
\hline \multirow{2}{*}{ LE } & RMSE & 34.3 & 32.3 & 32.3 & 28.5 & 26.5 & 38.5 & 35.5 & 32.9 & 36.1 & 32.4 \\
& D20: Mean est. damage[cm] & 56.40 & 52.14 & 44.43 & 50.69 & 28.47 & 58.38 & 52.94 & 55.69 & 55.33 & 62.37 \\
& D60: Mean est. damage[cm] & 46.99 & 72.16 & 59.80 & 52.44 & 56.62 & 78.79 & 74.91 & 60.41 & 82.85 & 64.50 \\
\hline
\end{tabular}

The sensors located in the TE also had a better performance for the quantification of the damage. The results show that the location of the sensor in the TE and LE influences the results. The sensors that fall under scenarios i) and ii) referred to in damage detection are the ones that present the best results. In TE, sensors 1, 2, 3, 4, and 8 had the best results. Sensor 10 had the worst result in the TE, which did not detect the damage well. The results of the LE sensors were not good, with sensors 4 and 5 that best quantified the damage, and these sensors were the ones that detected a trend in the progression of damage. The metamodel obtained by PC-Kriging presents promising results for the quantification of the damage to the DIs that present good detection of the damage or capture the damage progression trend.

\section{Conclusions}

This work approached the debonding area's quantification due to the debonding in wind turbine blades, using a new methodology considering the uncertainties and interpolation through a metamodel obtained by the Polynomial ChaosKriging (PC-Kriging) method. The metamodel obtained relates the damage indexes to trailing edge debonding. To obtain the damage indexes, the methodology presented in
García and Tcherniak (2019) was used. This methodology was applied to an SSP $34 \mathrm{~m}$ wind turbine blade, instrumented with one actuator, ten accelerometers in the trailing edge (TE), and ten in the leading edge (LE). It is observed that the accelerometers located in the TE detected the damage better than those located in the LE. The excellent performance of accelerometer 4 in the TE stands out, it is located close to the damage and far from the actuator. It is also noted that the damage introduced is located in the TE, where the accelerometers obtained more favorable results.

The PC-Kriging method was used to obtain a metamodel that relates the damage index with severity to quantify the trailing edge debonding. A trend curve was obtained for this relationship, considering a $95 \%$ confidence interval. The PCE method captures the computational model's global behavior, while the Kriging method, of the interpolation type, captures local variations. For this reason, PC-Kriging, which is the combination of the two methods, presents itself as a more robust method for obtaining metamodels.

In this study, the quantification of the damage using the PC-Kriging showed better TE's accelerometers' performances, highlighting the TE's accelerometer 4. With that, it can be concluded that the accelerometers that better detected the damage obtained better damage indexes, 

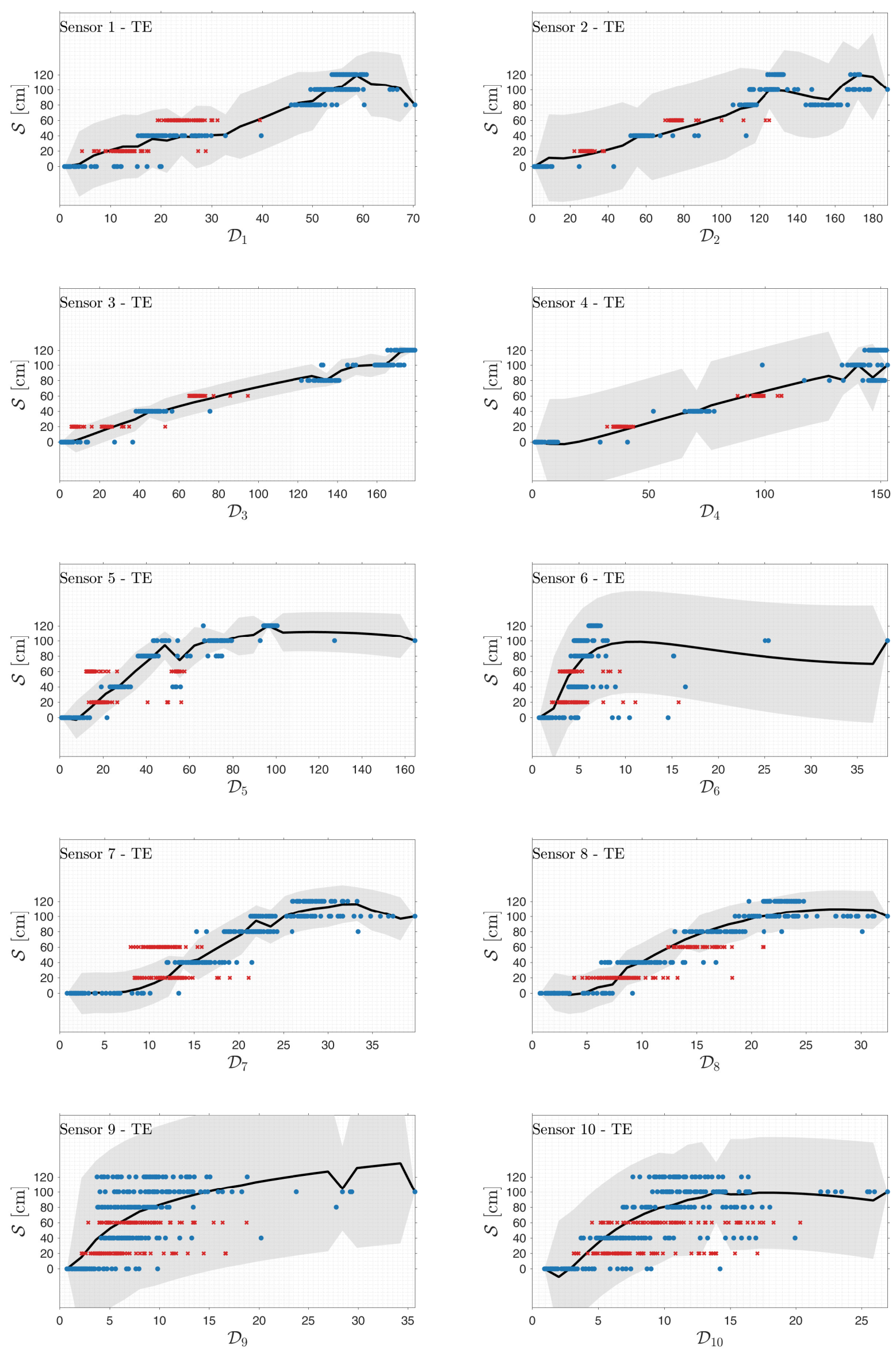

Figure 7. Damage severity $(\mathcal{S})$ by the damage index $\left(\mathcal{D}_{i}\right)$ for accelerometers 1 to 10 on the trailing edge (TE). The metamodel was trained using five conditions $(\mathbf{O})$ and tested with two conditions $(\mathbf{x})$. The bold line (_) corresponds to the trend mean and the gray-colored region $(\square)$ to the $95 \%$ of confidence interval. 

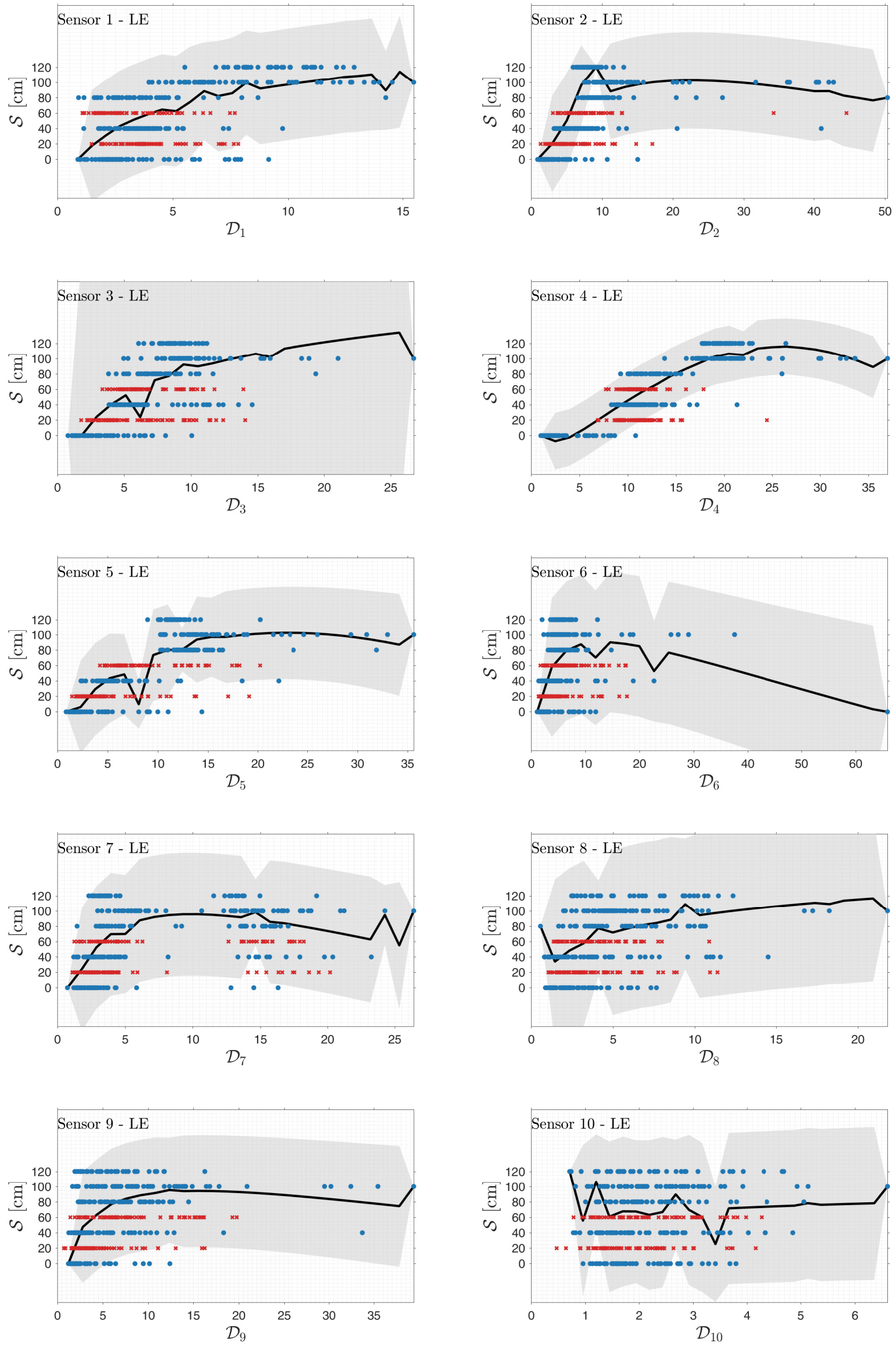

Figure 8. Damage severity $(\mathcal{S})$ by the damage index $\left(\mathcal{D}_{i}\right)$ for accelerometers 1 to 10 on the leading edge (LE). The metamodel was trained using five conditions $(\mathbf{O})$ and tested with two conditions $(\mathbf{x})$. The bold line $\left({ }_{-}\right)$corresponds to the trend mean and the gray-colored region ( $\square$ ) to the $95 \%$ of confidence interval. 

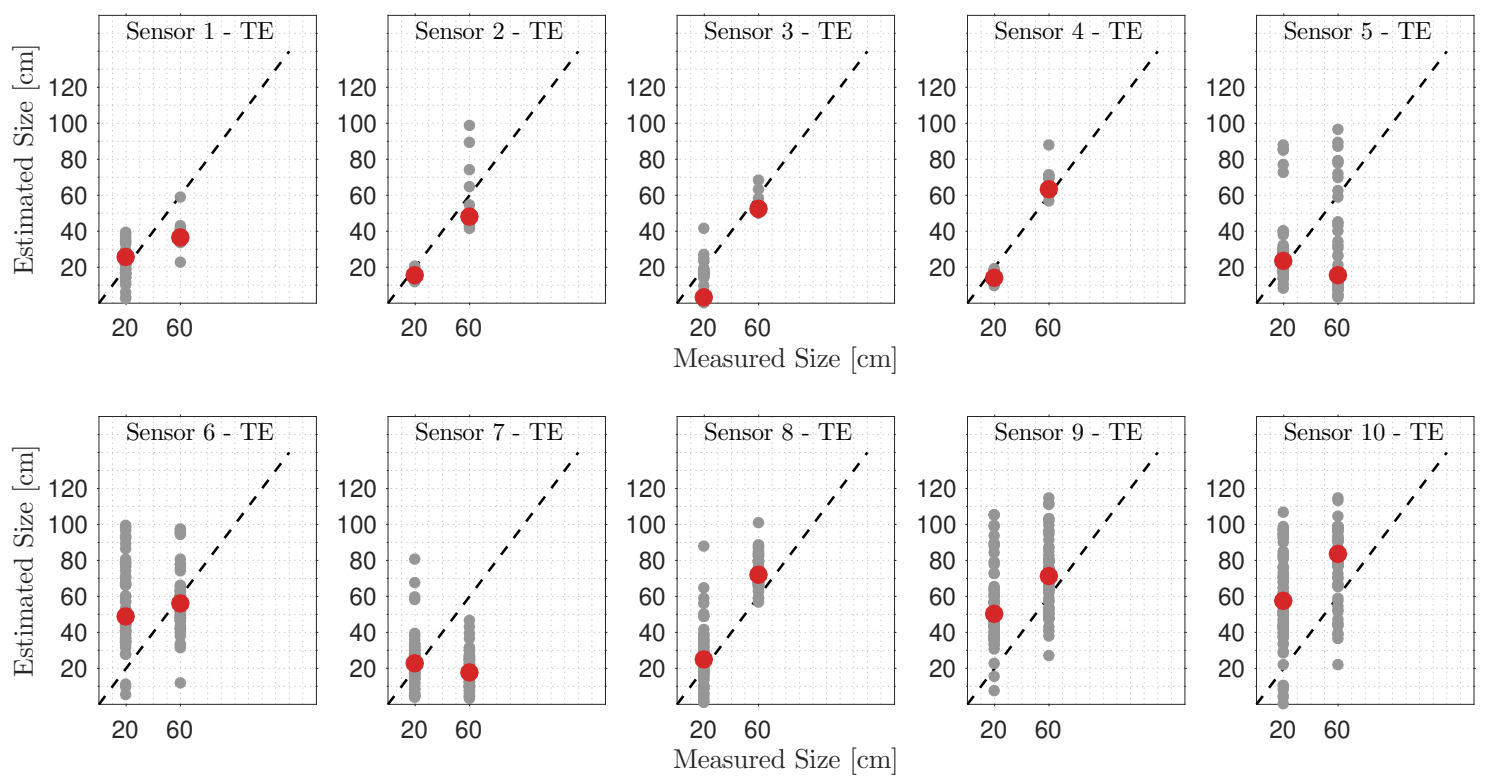

Figure 9. Validation of the estimated damage size using the PC-Kriging metamodel by the actual damage size for each sensor in the TE and test condition. The estimated damage size for all damage indexes $(\mathbf{O})$ and the mean of estimated damage size $(\mathbf{O})$ for each test condition.
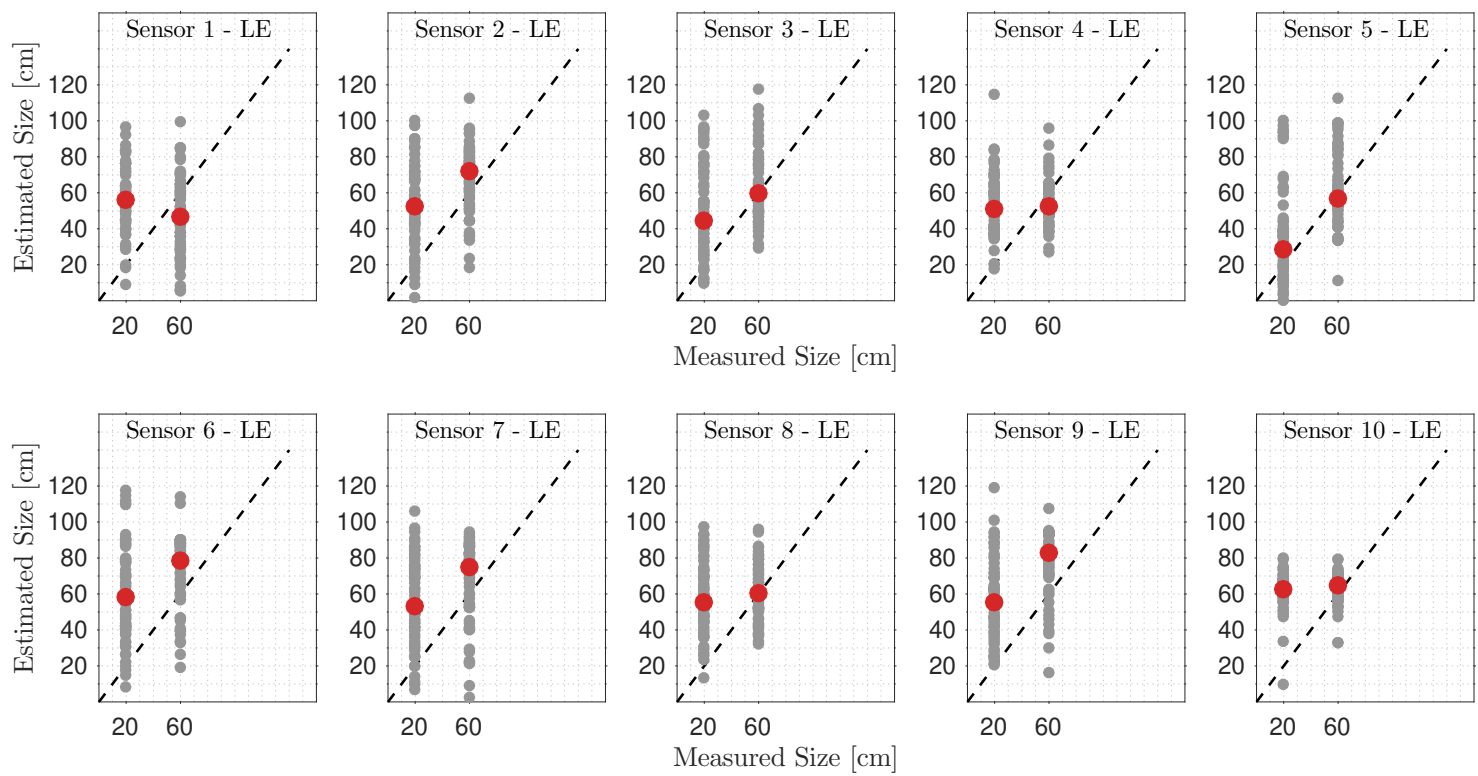

Figure 10. Validation of the estimated damage size using the PC-Kriging metamodel by the actual damage size for each sensor in the LE and test condition. The estimated damage size for all damage indexes (O) and the mean of estimated damage size (O) for each test condition.

and consequently resulted in a good performance for the quantification. It is also observed that the location of the accelerometers influences the results. The PC-Kriging trend curve, in general, managed to capture a monotonic increase in damage indexes, showing promising results for quantification. The advantages observed in the use of PC-Kriging were its simplicity and ease in constructing the metamodel since the toolbox UQLab is available, which allows an easy implementation of the method. It is also observed the low computational cost to obtain the results.
This study presents a contribution to the data-driven SHM methodology regarding the quantification of damages in mechanical structures, as it addresses the use of a method that is not yet widely explored in this area. This study collaborates with the development of research in damage quantification so that better results are always obtained, and thus, it is possible to apply in the industrial context and contribute to society. 


\section{Acknowledgments}

The authors of this work would like to acknowledge the generous input of Dr. Dmitri Tcherniak, from Brüel \& Kjær Sound and Vibration Measurements, who kindly provided the data from the experimental regime performed on the SSP $34 \mathrm{~m}$ wind turbine blade.

\section{Declaration of conflicting interests}

The authors declared no potential conflicts of interest concerning the research, authorship, and publication of this article.

\section{Funding}

The authors are thankful for the financial support provided by the São Paulo Research Foundation (FAPESP) grants 2017/155128, 2018/15671-1, 2019/11755-9 and 2019/19684-3; the Brazilian National Council for Scientific and Technological Development (CNPq/Brazil), grant number 306526/2019-0. We would also like to acknowledge the Carlos Chagas Filho Research Foundation of Rio de Janeiro State (FAPERJ) under grants 210.021/2018 and 211.037/2019, and Coordenação de Aperfeiçoamento de Pessoal de Nível Superior - Brasil (CAPES) - Finance Code 001.

\section{References}

Avendaño-Valencia LD, Barahona B, Hoelzl C and Chatzi E (2017) Operational regime clustering for the construction of PCE-based surrogates of operational wind turbines. 7th International Conference on Advances in Experimental Structural Engineering (7AESE) 17: 961-964.

Avendaño-Valencia LD, Chatzi E and Spiridonakos M (2015) Non-stationary random coefficient models for vibrationbased SHM in structures influenced by strong operational and environmental variability. International Workshop On Structural Health Monitoring DOI:10.12783/SHM2015/101.

Bogoevska S, Spiridonakos M, Chatzi E, Dumova-Jovanoska E and Höffer R (2017) A data-driven diagnostic framework for wind turbine structures: a holistic approach. Sensors (Basel) 17: 720 DOI:10.3390/s17040720.

Ciang CC, Lee JR and Bang HJ (2008) Structural health monitoring for a wind turbine system: a review of damage detection methods. Measurement Science and Technology 19(12): 122001. DOI:10.1088/0957-0233/19/12/122001.

Da Silva S, Paixão J, Rébillat $M$ and Mechbal N (2019) Data-driven autoregressive model identification for structural health monitoring in anisotropic composite plates. In: Ayech Benjeddou NM and Deü JF (eds.) IX ECCOMAS Thematic Conference on Smart Structures and Materials SMART 2019. International Centre for Numerical Methods in Engineering (CIMNE), Paris, França: Artes Gráficas Torres S.L, pp. 1213-1223.

Du X and Leifsson L (2020) Multifidelity modeling by polynomial chaos-based cokriging to enable efficient model-based reliability analysis of NDT systems. Journal of Nondestructive Evaluation 39. DOI:10.1007/s10921-020-0656-8.

Dubreuil S, Bartoli N, Gogu C, Lefebvre T and Colomer JM (2018) Extreme value oriented random field discretization based on an hybrid polynomial chaos expansion - kriging approach. Computer Methods in Applied Mechanics and Engineering 332: 540 - 571. DOI:10.1016/j.cma.2018.01.009.
García D and Tcherniak D (2019) An experimental study on the data-driven structural health monitoring of large wind turbine blades using a single accelerometer and actuator. Mechanical Systems and Signal Processing 127: 102 - 119. DOI:10.1016/ j.ymssp.2019.02.062.

García D and Trendafilova I (2014) A multivariate data analysis approach towards vibration analysis and vibration-based damage assessment:: Application for delamination detection in a composite beam. Journal of Sound and Vibration 333(25): 7036 - 7050. DOI:10.1016/j.jsv.2014.08.014.

Ghanem R, Owhadi H and Higdon D (2017) Handbook of Uncertainty Quantification. Springer International Publishing. DOI:10.1007/978-3-319-12385-1.

Ghanem R and Spanos PD (1990) Polynomial chaos in stochastic finite elements. ASME. J. Appl. Mech. 57: 197-202. DOI: $10.1115 / 1.2888303$.

Ghanem RG and Spanos PD (1991) Stochastic Finite Elements: A Spectral Approach. Berlin, Heidelberg: Springer-Verlag.

Hernandez Crespo B (2016) Damage Sensing in Blades. Cham: Springer International Publishing, pp. 25-52.

Kersaudy P, Sudret B, Varsier N, Picon O and Wiart J (2015) A new surrogate modeling technique combining kriging and polynomial chaos expansions - application to uncertainty analysis in computational dosimetry. Journal of Computational Physics 286: 103 - 117. DOI:10.1016/j.jcp.2015.01.034.

Larrosa C, Lonkar K and Chang FK (2014) In situ damage classification for composite laminates using Gaussian discriminant analysis. Structural Health Monitoring 13(2): 190-204. DOI: 10.1177/1475921713517288.

Lataniotis C, Marelli S and Sudret B (2015) UQLAB User Manual - Kriging (Gaussian process modelling). DOI:10.13140/RG.2. 1.4827.3120.

Love B (2002) Comparing supervised and unsupervised category learning. Psychonomic bulletin review 9: 829-835. DOI: 10.3758/BF03196342.

Maes K, Iliopoulos A, Weijtjens W, Devriendt C and Lombaert G (2016) Dynamic strain estimation for fatigue assessment of an offshore monopile wind turbine using filtering and modal expansion algorithms. Mechanical Systems and Signal Processing 76-77: 592 - 611. DOI:10.1016/j.ymssp.2016.01. 004.

Marelli S and Sudret B (2014) UQLab: a framework for uncertainty quantification in MATLAB. In: Proc. 2nd Int. Conf. on Vulnerability, Risk Analysis and Management (ICVRAM2014), Liverpool, United Kingdom.

Marelli S and Sudret B (2015) UQLab user manual - Polynomial chaos expansions. DOI:10.13140/RG.2.1.3778.7366. URL https://www.uqlab.com/pce-user-manual.

Montesano J, Chu H and Singh CV (2016) Development of a physics-based multi-scale progressive damage model for assessing the durability of wind turbine blades. Composite Structures 141: 50 - 62. DOI:10.1016/j.compstruct.2016.01. 011.

Nielsen M, Roczek-Sieradzan A, Nielsen PH, Berring P, Sieradzan T, Roudnitski V, Bitsche RD, Knudsen HW, Rasmussen AB, Rasmussen JJA, Uldahl U, Andrlová Z, Branner K, Bak C, Kallesøe BS, McGugan M, Lagerbon M, Wedel-Heinen J, Lindby T, Riber HJ and Jensen C (2010) Full scale test ssp $34 \mathrm{~m}$ blade, edgewise loading ltt. extreme load and poc_inve data report. 
Paixão J, Da Silva S and Figueiredo E (2020) Damage quantification in composite structures using autoregressive models. In: Wahab MA (ed.) Proceedings of the 13th International Conference on Damage Assessment of Structures. Singapore: Springer Singapore, pp. 804-815. DOI:10.1007/ 978-981-13-8331-1_63.

Rytter A (1993) Vibrational Based Inspection of Civil Engineering Structures. PhD Thesis, Denmark. Ph.D.-Thesis defended publicly at the University of Aalborg, April 20, 1993 PDF for print: $206 \mathrm{pp}$.

Schöbi R, Kersaudy P, Sudret B and Wiart J (2014) Combining Polynomial Chaos Expansions and Kriging. Research report, ETH Zurich, Switzerland ; Orange Labs research.

Schöbi R, Marelli S and Sudret B (2017) UQLab User Manual PC-Kriging. DOI:10.13140/RG.2.1.4827.3120.

Schöbi R and Sudret B (2014) Pc-kriging: A new metamodelling method combining polynomial chaos expansions and kriging. Rouen, France.

Schöbi R, Sudret B and Marelli S (2016) Rare event estimation using polynomial-chaos kriging. ASCE-ASME Journal of Risk and Uncertainty in Engineering Systems, Part A: Civil Engineering 500: D4016002. DOI:10.1061/AJRUA6.0000870.

Schöbi R, Sudret B and Wiart J (2015) Polynomial-chaos-based kriging. International Journal of Uncertainty Quantification 5. DOI:10.1615/Int.J.UncertaintyQuantification.2015012467.

Soize C and Orcesi A (2020) Machine learning for detecting structural changes from dynamic monitoring using the probabilistic learning on manifolds. Structure and Infrastructure Engineering 0(0): 1-13. DOI:10.1080/15732479.2020.1811991.

Tcherniak D and Mølgaard LL (2015) Vibration-based SHM system: Application to wind turbine blades. Journal of Physics: Conference Series 628: 012072. DOI:10.1088/1742-6596/628/ $1 / 012072$.

Ulriksen MD, Tcherniak D, Kirkegaard PH and Damkilde L (2016) Operational modal analysis and wavelet transformation for damage identification in wind turbine blades. Structural Health Monitoring 15(4): 381-388. DOI:10.1177/ 1475921715586623.

Xiu D (2010) Numerical Methods for Stochastic Computations: A Spectral Method Approach. New Jersey, USA.

Xiu D and Karniadakis G (2002) The Wiener-Askey polynomial chaos for stochastic differential equations. SIAM J. Sci. Comput. 24: 619-644. DOI:10.1137/S1064827501387826. 